\title{
The scalar pion form factor with Wilson fermions
}

\author{
Vera Gülpers $^{* 1,2}$, Georg von Hippel ${ }^{1}$, Hartmut Wittig ${ }^{1,2}$ \\ ${ }^{1}$ PRISMA Cluster of Excellence, Institut für Kernphysik, Johannes Gutenberg Universität Mainz, \\ 55099 Mainz, Germany \\ ${ }^{2}$ Helmholtz Institute Mainz, Johannes Gutenberg Universität Mainz, 55099 Mainz, Germany \\ E-mail: guelpersakph.uni-mainz.de
}

\begin{abstract}
We calculate the scalar form factor of the Pion in $N_{f}=2 \mathrm{QCD}$ with $O(a)$ improved Wilson fermions including both the connected and the disconnected contributions, where the latter can be estimated using stochastic sources. We will show that the statistical error of this estimation can be reduced using a generalized hopping parameter expansion. To extract the scalar form factor we build suitable ratios of three- and two-point functions. We report on our attempts to calculate the scalar radius from the form factor.
\end{abstract}

The 30th International Symposium on Lattice Field Theory

June 24 - 29, 2012

Cairns, Australia

${ }^{*}$ Speaker. 


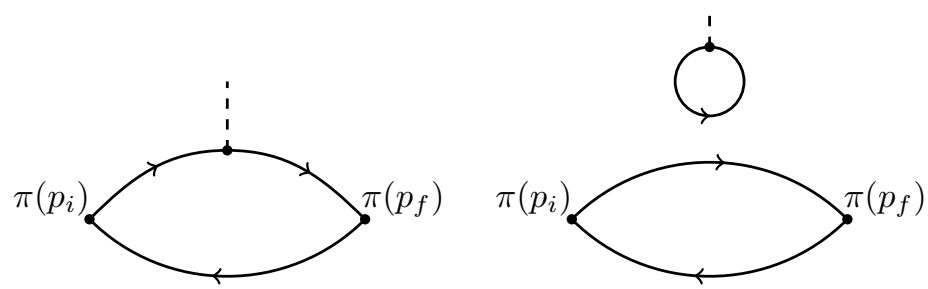

Figure 1: connected (left) and disconnected (right) contribution to the scalar form factor of the pion

\section{Introduction}

The scalar form factor of the pion describes the coupling of a charged pion to a scalar particle. It is not yet possible to measure the scalar form factor directly in an experiment, but it can be calculated using lattice QCD. The scalar form factor can be defined as

$$
F_{S}\left(Q^{2}\right) \equiv\left\langle\pi^{+}\left(p_{f}\right)\left|m_{d} \bar{d} d+m_{u} \bar{u} u\right| \pi^{+}\left(p_{i}\right)\right\rangle
$$

with the momentum transfer $Q^{2}=-q^{2}=-\left(p_{f}-p_{i}\right)^{2}$. From the scalar form factor the scalar radius

$$
\left\langle r^{2}\right\rangle_{s}=-\left.\frac{6}{F_{S}(0)} \frac{\partial F_{s}\left(Q^{2}\right)}{\partial Q^{2}}\right|_{Q^{2}=0}
$$

can be determined, in analogy with the charge radius in the vector case. Using chiral perturbation theory $(\chi \mathrm{PT})$ the scalar radius depends on only one low energy constant $\bar{l}_{4}$ at one loop level [1]. Additionally it is possible to relate the scalar radius to the $\pi \pi$-scattering amplitude. In [2] a scalar radius $\left\langle r^{2}\right\rangle_{s}=0.61 \pm 0.04 \mathrm{fm}^{2}$ was obtained using $\pi \pi$-scattering data.

When performing the Wick contractions for the matrix element in (1.1), two contributions are obtained, a quark-connected and a quark-disconnected one. The latter is computationally more demanding, because the calculation of the disconnected loop requires knowledge of the all-to-all propagator which can be estimated using stochastic sources. In order to keep the required computer time at a minimum, it is important to use a small number of stochastic sources. However, the fewer sources used the larger is the error of the disconnected contribution. This error can be reduced using a generalized hopping parameter expansion (cf section 2).

Our calculations are done with non-perturbatively $O(a)$-improved Wilson fermions and the Wilson gauge action with $N_{f}=2$ using the CLS configurations [3].

\section{The generalized Hopping Parameter Expansion}

The $O(a)$-improved Wilson-Dirac operator has the form [4]

$$
D_{S W}=\frac{1}{2 \kappa} \mathbb{1}+c_{S W} B-\frac{1}{2} H
$$

with the hopping parameter $\kappa$, the $O(a)$-improvement term $c_{S W} B$ and the hopping matrix $H$, which contains all contributions that couple neighboring lattice points. Equation (2.1) can be rewritten as

$$
D_{S W}=A-\frac{1}{2} H=A\left(\mathbb{1}-\frac{1}{2} A^{-1} H\right)
$$




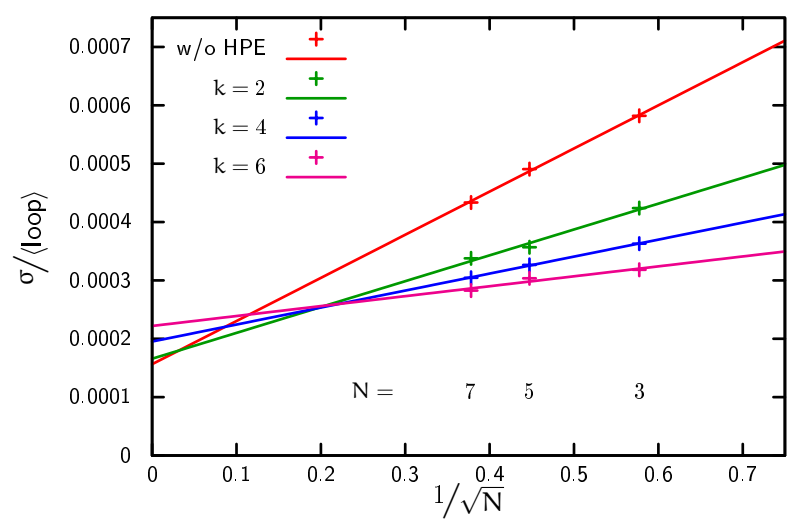

Figure 2: standard deviation of the gauge mean of the loop

where $A=(2 \kappa)^{-1} \mathbb{1}+c_{S W} B$. To obtain the propagator, i.e. the inverse of $D_{S W}$, a geometric series expansion in $\kappa$ can be performed,

$$
D_{S W}^{-1}=\sum_{i=0}^{k-1}\left(\frac{1}{2} A^{-1} H\right)^{i} A^{-1}+\left(\frac{1}{2} A^{-1} H\right)^{k} D_{S W}^{-1} .
$$

Here the inverse of the matrix $A$ is needed. For $c_{S W}=0$ the inverse $A^{-1}$ is trivial and (2.3) is a series expansion in $\kappa$, called hopping parameter expansion (HPE) [5]. With $\mathscr{O}(a)$-improvement $\left(c_{S W} \neq 0\right)$ a generalized HPE has to be applied. The local form of the improvement term implies that the matrix $A$ is block diagonal, such that two $6 \times 6$ matrices have to be inverted for each lattice point, which is still comparatively cheap in computational costs.

The remaining inverse $D_{S W}^{-1}$ on the right-hand side of (2.3) can be calculated with $N$ stochastic sources $\left|\eta_{i}\right\rangle$ as follows [5]

$$
D_{S W}\left|s_{i}\right\rangle=\left|\eta_{i}\right\rangle \quad D_{S W}^{-1}=\frac{1}{N} \sum_{i}\left|s_{i}\right\rangle\left\langle\eta_{i}\right|
$$

Therefore one has two parameters to tune - the number of stochastic sources $N$ and the order $k$ of the HPE. In figure 2 the standard deviation divided by the gauge mean of the scalar loop

$$
\langle\text { loop }\rangle=\left\langle\sum_{\vec{x}} \operatorname{Tr}\left(D^{-1}(x, x)\right)\right\rangle_{G}
$$

is plotted against $\sqrt{N}^{-1}$ without HPE and with $k=2,4,6$. One can clearly see that the noise is reduced when applying more terms in the HPE. One finds the expected linear behavior in $\sqrt{N}^{-1}$, thus the $y$-axis intercept indicates the remaining gauge noise.

For the calculation of the scalar form factor we use $N=3$ sources and $k=6$ terms for the generalized hopping parameter expansion, which gives a good balance between the accuracy of the calculation and the computer time needed. 


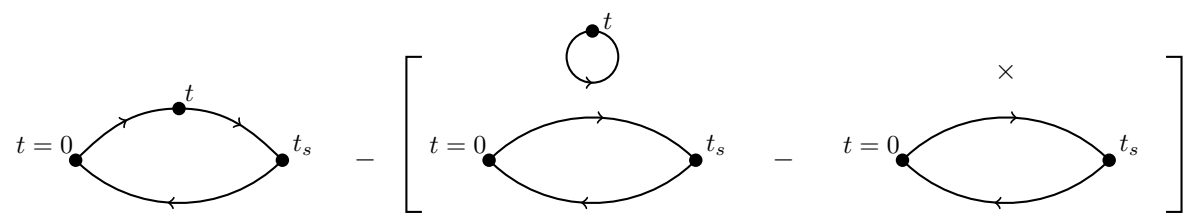

Figure 3: The three contributions to the three-point function. The connected on the left, the disconnected with subtracted vacuum on the right.

\section{Extracting the Form Factor}

\subsection{Two- and three point functions}

The scalar form factor of the pion can be extracted using two- and three-point functions. The pseudoscalar two-point function with momentum $\mathbf{p}$ is expected to behave like

$$
C_{2 \mathrm{pt}}\left(t_{s}, \mathbf{p}\right)=\left\langle\phi\left(t_{s}, \mathbf{p}\right) \phi(0, \mathbf{p})\right\rangle \sim \frac{Z_{p}^{2}}{2 E(\mathbf{p})}\left[e^{-E_{\pi} t_{s}}+e^{-E_{\pi}\left(T-t_{s}\right)}\right] .
$$

In expression (3.1) excited states are neglected and $Z_{p}=|\langle\pi(\mathbf{p})|\phi(0)| 0\rangle|$ is the overlap of a pion with the pseudoscalar field $\phi=\bar{q} \gamma_{5} q$. For our calculations we put the pion source at $t=0$ and the pion sink at $t_{s}$. The two summands in (3.1) correspond to the forward and the backward propagating pion due to the periodic boundary conditions used in our simulations.

Now the scalar operator $\mathscr{O}=\bar{q} q$ is inserted at a time $t$ with $0<t<t_{s}$. The corresponding three-point function with subtracted vacuum contributions has the behavior

$$
\begin{aligned}
C_{3 \mathrm{pt}}\left(t, t_{s}, \mathbf{p}_{i}, \mathbf{p}_{f}\right) & =\left\langle\phi\left(t_{s}, \mathbf{p}_{f}\right) \mathscr{O}(t, \mathbf{q}) \phi\left(0, \mathbf{p}_{i}\right)\right\rangle \\
& \sim \frac{Z_{p_{i}} Z_{p_{f}}}{4 E_{\pi}\left(\mathbf{p}_{i}\right) E_{\pi}\left(\mathbf{p}_{f}\right)}\left\langle\pi\left(\mathbf{p}_{f}\right)|\mathscr{O}(\mathbf{q})| \pi\left(\mathbf{p}_{i}\right)\right\rangle e^{-\left(t_{s}-t\right) E_{\pi}\left(\mathbf{p}_{f}\right)} e^{-t E_{\pi}\left(\mathbf{p}_{i}\right)}
\end{aligned}
$$

with the momentum transfer $q^{2}=\left(p_{f}-p_{i}\right)^{2}$. As for the two-point function, excited states have been neglected. The contributing diagrams are shown in figure 3 .

The matrix element $\left\langle\pi\left(\mathbf{p}_{f}\right)|\mathscr{O}(\mathbf{q})| \pi\left(\mathbf{p}_{i}\right)\right\rangle$ in (3.2) now has to be extracted from the two- and three-point functions.

\subsection{Extracting the form factor}

The form factor can be extracted from the three- and two-point data by building a ratio like [6]

$$
\begin{aligned}
& R=\sqrt{\frac{C_{3 \mathrm{pt}}\left(t, t_{s}, \mathbf{p}_{i}, \mathbf{p}_{f}\right) C_{3 \mathrm{pt}}\left(t, t_{s}, \mathbf{p}_{f}, \mathbf{p}_{i}\right)}{C_{2 \mathrm{pt}}\left(t_{s}, \mathbf{p}_{i}\right) C_{2 \mathrm{pt}}\left(t_{s}, \mathbf{p}_{f}\right)}} \\
& \sim \frac{\left\langle\pi\left(\mathbf{p}_{f}\right)|\mathscr{O}(\mathbf{q})| \pi\left(\mathbf{p}_{i}\right)\right\rangle}{2 \sqrt{E_{\pi}\left(\mathbf{p}_{i}\right) E_{\pi}\left(\mathbf{p}_{f}\right)}} \sqrt{\frac{e^{-E_{\pi}\left(\mathbf{p}_{i}\right) t_{s}} e^{-E_{\pi}\left(\mathbf{p}_{f}\right) t_{s}}}{\left(e^{-E_{\pi}\left(\mathbf{p}_{i}\right) t_{s}}+e^{-E_{\pi}\left(\mathbf{p}_{i}\right)\left(T-t_{s}\right)}\right) \cdot\left(e^{-E_{\pi}\left(\mathbf{p}_{f}\right) t_{s}}+e^{-E_{\pi}\left(\mathbf{p}_{f}\right)\left(T-t_{s}\right)}\right)}} .
\end{aligned}
$$

As one can see, all undesired factors of $Z_{p}$ cancel in (3.3). Neglecting excited states, the ratio is expected to be independent of the operator insertion time $t$. Only a simple dependence on $t_{s}$ remains which is parameter-free, since $E_{\pi}(\mathbf{p})$ can be determined from the two-point functions. This $t_{s}$ dependence is an effect of the finite lattice volume, since the two-point function has a 


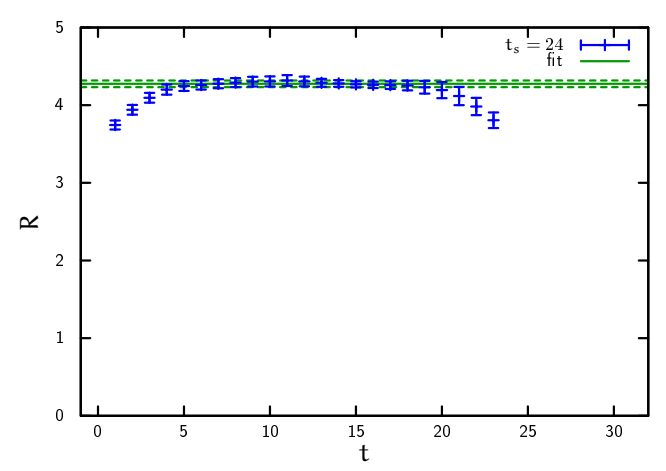

(a) connected: fitrange $t$ from 5 to 19

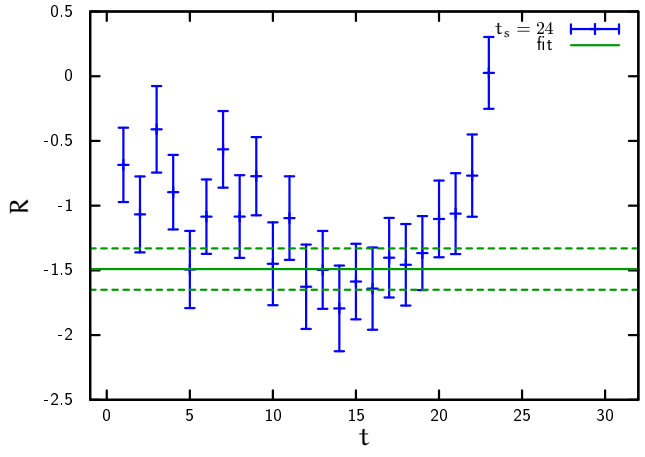

(b) disconnected: fitrange $t$ from 10 to 19

Figure 4: Ratios for $\mathbf{p}_{i}=\mathbf{p}_{f}=0$ plotted against $t$ for fixed $t_{s}=24$

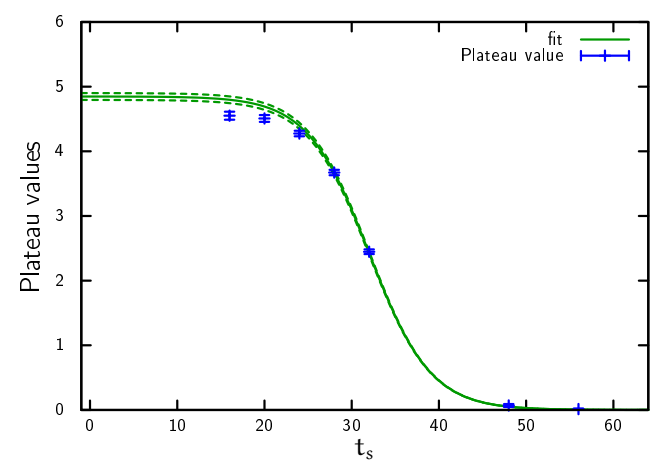

(a) connected

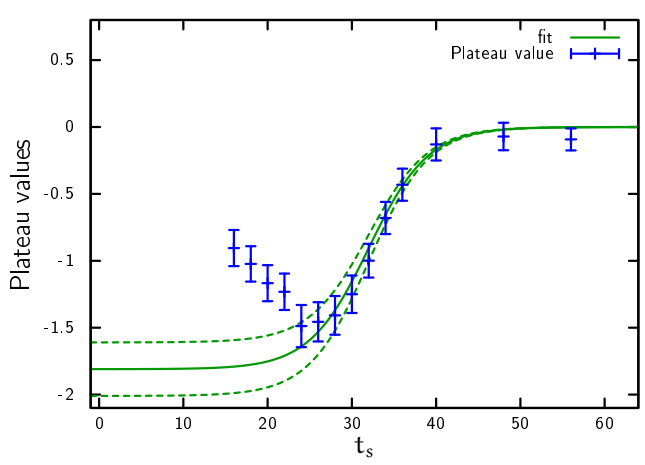

(b) disconnected

Figure 5: Plateau values for $\mathbf{p}_{i}=\mathbf{p}_{f}=0$ plotted against $t_{s}$

backward propagating part as indicated in (3.1) when using periodic boundary conditions. For an infinite time extent $T \rightarrow \infty$, the $t_{s}$ dependence vanishes.

The calculation of the connected three-point function is performed with the extended propagator method [7] and the loop in the disconnected contribution is estimated with stochastic sources and the generalized HPE as described above.

Figures 4 to 6 show the results obtained from a $64 \times 32^{3}$ lattice with a lattice spacing of $0.063 \mathrm{fm}$ and $m_{\pi}=443 \mathrm{MeV}$, with high statistics of 1000 configurations.

In figure 4 the ratios for $\mathbf{p}_{i}=\mathbf{p}_{f}=0$, i.e. vanishing momentum transfer, are plotted against the time $t$ of the operator insertion for a fixed $t_{s}=24$ as an example. In both the connected and the disconnected part the data form a plateau and a constant is fitted to the plateau region. The deviation from the plateau value near $t=0$ and $t=t_{s}$ is due to excited state contributions which have not been taken into account in (3.3). These ratios have been calculated for different values of $t_{s}$ and the corresponding plateau values are plotted in figure 5. One can clearly see the expected $t_{s}$ dependence due to finite volume effects. The deviation from the expected curvature at the smallest $t_{s}$ indicate the contamination with excited states. A function of the form (3.3) can be fitted to the data to obtain the connected and the disconnected contribution to the scalar form factor. To avoid 


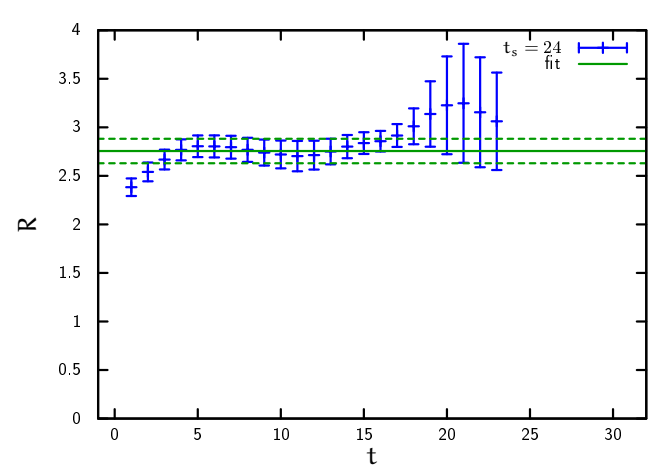

(a) ratios for fixed $t_{s}=24$

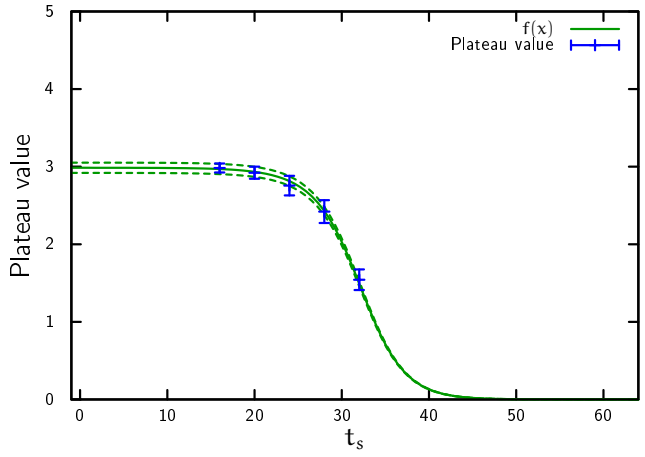

(b) Plateau values plotted against $t_{s}$

Figure 6: Results for the connected part with $q^{2}=-0.278 \mathrm{GeV}^{2}$

systematics from excited state contributions all points with $t_{s}<24$ have been excluded.

In figure 6 the results for the connected part with the smallest momentum transfer available are shown. We have inserted the momenta via Fourier transformation, thus the smallest possible momentum is $|\mathbf{p}|=2 \pi / L$. As for non-vanishing momentum transfer the plateau values obtained for the different $t_{s}$ can be plotted against $t_{s}$ and a function of the form (3.3) can be fitted to the data (figure 6b).

For all ensembles we looked at so far, the smallest non-vanishing $q^{2}$ is quite large and we do not see a signal for the disconnected contribution which is different from zero within the errors. This has to be investigated using ensembles with larger volumes and thus smaller $q^{2}$.

\subsection{A few words on Renormalization}

Since our simulations are performed using $O(a)$-improved Wilson fermions, chiral symmetry is explicitly broken. Therefore the scalar operator mixes with the identity and one obtains an additive renormalization besides the multiplicative one.

$$
\left\langle\mathscr{O}^{R}\right\rangle=Z_{s}\left\langle\mathscr{O}-b_{0}\right\rangle
$$

However, the vacuum contributions have to be subtracted as illustrated in figure 3. When subtracting the vacuum also the additive renormalization is subtracted such that the form factor is independent of $b_{0}$.

While $Z_{s}$ is required to obtain the correctly normalized scalar form factor, it drops out in the determination of the scalar radius ( $\mathrm{cf}(1.2)$ ). All form factor data shown above are not renormalized, since $Z_{S}$ has not been determined yet.

\section{The scalar Radius}

To determine the scalar radius $\left\langle r^{2}\right\rangle_{s}$ it is convenient to parametrize the form factor like

$$
F_{S}\left(Q^{2}\right)=F_{s}(0)\left(1-\frac{1}{6}\left\langle r^{2}\right\rangle_{s} Q^{2}+\mathscr{O}\left(Q^{4}\right)\right) \text {. }
$$


(4.1) implies that a rough estimate for $\left\langle r^{2}\right\rangle_{s}$ is possible with the form factor data from $Q^{2}=0$ and one other value of $Q^{2}$. Since we are not yet able to resolve a signal for the disconnected part at non-vanishing momentum transfer, we currently restrict the calculation of the scalar radius to the connected part of the form factor. For the used ensemble we obtain $\left\langle r^{2}\right\rangle_{s}^{\text {con }}=0.164 \pm 0.018 \mathrm{fm}^{2}$. A recent calculation in $\chi \mathrm{PT}$ [8] has shown, that the disconnected contribution to the scalar radius is of the same order as the connected one. While for $Q^{2}=0$ we obtain a significant contribution to the scalar form factor from the disconnected part, the disconnected contribution for $Q^{2} \neq 0$ is consistent with zero. Assuming the disconnected part to be close to zero at the smallest nonvanishing momentum transfer we obtain a rough estimate $\left\langle r^{2}\right\rangle_{s} \approx 0.35 \mathrm{fm}^{2}$.

\section{Conclusion}

We have shown that the calculation of disconnected contribution to the scalar form factor of the pion works for $Q^{2}=0$ using Wilson fermions. The generalized hopping parameter expansion is a useful tool to reduce the statistical error of the all-to-all propagator calculated with stochastic sources. With the high statistics used for our calculation we are able to resolve the exited state contributions for both the connected and the disconnected contributions.

Our data indicate that the disconnected contribution to the scalar radius is significant. The smallest non-zero $Q^{2}$ might still be too large to observe a non-vanishing contribution from disconnected diagrams. If true this would imply a very strong suppression of the scalar form factor as $Q^{2}$ increases. This requires further investigations at smaller pion masses and larger volumes, which are currently under way.

\section{Acknowledgments}

Our calculations were performed on the dedicated QCD platform "Wilson" at the Institute for Nuclear Physics, University of Mainz.

\section{References}

[1] J. Gasser and H. Leutwyler, Ann. Phys. 158, (1984) 142-210

[2] G. Colangelo, J. Gasser and H. Leutwyler, Nucl. Phys. B603, (2001) 125-179, [hep-ph/0103088]

[3] https://twiki.cern.ch/twiki/bin/view/CLS/WebIntro

[4] B. Sheikholeslami and R. Wohlert, Nucl. Phys. B259, (1985) 572-596

[5] G. S. Bali, S. Collins and A. Schäfer, Comput. Phys. Commun. 181, (2010) 1570-1583, [arXiv:0910.2970]

[6] P. A. Boyle et al, JHEP 0705, (2007) 016, [hep-lat/0703005]

[7] G. Martinelli and C. T. Sachrajda, Nucl. Phys. B316, (1989) 355-372

[8] A. Jüttner, JHEP 01, (2012) 007, [arXiv:1110.4859] 